Оригинальная статья / Original article

УДК 620.197:669.017

DOI: https://doi.org/10.21285/2227-2925-2021-11-2-187-194

\author{
Анодное поведение сплава Zn0.5Al, \\ легированного молибденом, \\ в кислых, нейтральных и щелочных средах \\ () 3.Р. Обидов, П.Р. Иброхимов, Ф.А. Рахимов, И.Н. Ганиев \\ Институт химии им. В.И. Никитина АН Республики Таджикистан, \\ г. Душанбе, Республика Таджикистан
}

\begin{abstract}
Резюме: Приведены результаты потенциодинамического исследования анодного поведения цинкового сплава Zn0,5Al, легированного молибденом в кислых $(0,1 \mathrm{M}, \mathrm{pH}=1 ; 0,01 \mathrm{M}, \mathrm{pH}=2 ; 0,001 \mathrm{M}$, pH = 3), нейтральных (0,03, 0,3, 3\%; $\mathrm{pH}=7)$ и щелочных $(0,001 \mathrm{M}, \mathrm{pH}=10 ; 0,01 \mathrm{M}, \mathrm{pH}=11 ; 0,1 \mathrm{M}$, $\mathrm{pH}=12)$ средах электролитов $\mathrm{HCl}, \mathrm{NaCl}$ и $\mathrm{NaOH}$. В потенциодинамическом режиме со скоростью развертки электродного потенциала 2 мB/с выявлено, что для всех образцов сплавов Zno.5Al-Mo, содержащих от 0,01 до 1,0\% масс. молибдена, наблюдается смещение потенциалов коррозии, питтингообразования и репассивации, причем в кислых и щелочных средах происходит смещение в область отрицательных значений, в нейтральной среде - в область положительных значений. Установлено, что при повышении концентрации электролитов рассматриваемые потенциалы значительно смещаются в область отрицательных значений во всех средах - кислых, нейтральных и щелочных. Данная зависимость связана с особенностями механизма анодного растворения сплавов по мере фрормирования оксидной пленки на их поверхности. Показана важная роль зависимости стационарного потенциала свободной коррозии сплавов от времени в установлении пассивности поверхности в кислых, нейтральных и щелочных средах. Определено, что цинковые сплавы, легированные молибденом, устойчивы к питтинговой коррозии во всех исследованных средах. Особенно высокой оказывается устойчивость в кислой $(0,001 \mathrm{M})$, нейтральной $(0,03 \%)$ и щелочной (0,001 M) средах электролитов $\mathrm{HCl}, \mathrm{NaCl}$ и $\mathrm{NaOH}$. Показано благоприятное влияние молибдена на анодное поведение цинкового сплава Zno.5Al и повышение коррозионной стойкости сплавов в целом. Скорость коррозии легированных (0,01-1,0\% масс.) молибденом сплавов в 2-2,5 раза меньше, чем у нелегированного сплава Zno.5Al. Предложенные составы сплавов Zn0.5Al-Mo могут использоваться в качестве протекторов для защиты от коррозии стальных изделий $и$ конструкций.
\end{abstract}

Ключевые слова: сплав Zno.5Al с молибденом; кислая, нейтральная и щелочная среды; потенциалы коррозии и питтингообразования; потенциодинамическое исследование; скорость коррозии; анодное поведение сплавов

Для цитирования: Обидов З.Р., Иброхимов П.Р., Рахимов Ф.А., Ганиев И.Н. Анодное поведение сплава Zn0.5Al, легированного молибденом, в кислых, нейтральных и щелочных средах. Известия вузов. Прикладная химия и биотехнология. 2021. Т. 11. N 2. C. 187-194. https://doi.org/10.21285/22272925-2021-11-2-187-194

\title{
Anodic behaviour of Zn0.5Al doped with molybdenum in acidic, neutral and alkaline media
}

\section{Ziyodullo R. Obidov, Pairav R. Ibrohimov, Firuz A. Rahimov, Izatullo N. Ganiev}

V.I. Nikitin Institute of Chemistry, National Academy of Sciences of Tajikistan, Dushanbe, Republic of Tajikistan

Abstract: The paper presents the results of a potentiodynamic study of the anodic behaviour of Zno.5Al doped with molybdenum in the acidic $(0.1 \mathrm{M}, \mathrm{pH}=1 ; 0.01 \mathrm{M}, \mathrm{pH}=2 ; 0.001 \mathrm{M}, \mathrm{pH}=3)$, neutral (0.03, 0.3, $3 \%, p H=7)$ and alkaline $(0.001 \mathrm{M}, \mathrm{pH}=10 ; 0.01 \mathrm{M}, \mathrm{pH}=11 ; 0.1 \mathrm{M}, \mathrm{pH}=12)$ media of $\mathrm{HCl}, \mathrm{NaCl}$ and $\mathrm{NaOH}$ electrolytes. In the potentiodynamic mode with an electrode potential sweep rate of $2 \mathrm{mV} / \mathrm{s}$, all Zn0.5Al-Mo samples containing from 0.01 to 1.0 wt\% of molybdenum demonstrated a shift in the potentials of corrosion, pitting formation and repassivation. These potentials shift towards negative values in acidic and alkaline media, while shifting to positive values in a neutral medium. It was established that an increase in 
Обидов З.Р., Иброхимов П.Р., Рахимов Ф.А., Ганиев И.Н. Анодное поведение сплава Zn0.5Al, ... Obidov Z.R., Ibrohimov P.R., Rahimov F.A., Ganiev I.N. Anodic behaviour of Zn0.5Al ...

the concentration of electrolytes led to a shift of all the considered potentials towards negative values in all media - acidic, neutral and alkaline. This dependence is associated with the specific features of the process of anodic dissolution of alloys during the formation of an oxide film on their surface. The significance of the dependence of the stationary potential of free corrosion of alloys on time for establishing the passivity of surfaces in acidic, neutral and alkaline media was shown. It was determined that zinc alloys doped with molybdenum are resistant to pitting corrosion in all the investigated media. This resistivity is particularly high in acidic $(0.001 \mathrm{M})$, neutral $(0.03 \%)$ and alkaline $(0.001 \mathrm{M})$ media of $\mathrm{HCl}, \mathrm{NaCl}$ and $\mathrm{NaOH}$ electrolytes. The favourable effect of molybdenum on both the anodic behaviour of Zn0.5Al and the overall increase in the corrosion resistance of alloys was demonstrated. In comparison with undoped $\mathrm{ZnO} .5 \mathrm{Al}$ alloys, the corrosion rate of alloys doped with molybdenum (0.01-1.0 wt\%) is 2-2.5 times lower. The proposed compositions of Zn0.5Al-Mo alloys can be used as noncorrosive coatings for steel products.

Keywords: molybdenum-doped Zn0.5Al alloys; acidic, neutral and alkaline medium; corrosion and pitting potential; potentiodynamic study; corrosion rate; anodic behaviour of alloys

For citation: Obidov ZR, Ibrohimov PR, Rahimov FA, Ganiev IN. Anodic behaviour of Zn0.5Al doped with molybdenum in acidic, neutral and alkaline media. Izvestiya Vuzov. Prikladnaya Khimiya i Biotekhnologiya = Proceedings of Universities. Applied Chemistry and Biotechnology. 2021;11(2):187-194. (In Russian) https://doi.org/10.21285/2227-2925-2021-11-2-187-194

\section{ВВЕДЕНИЕ}

Цинк наиболее часто используется в электрохимических производствах металлопокрытий. До 40\% мировых запасов цинка расходуется для защиты металлоконструкций от коррозии. Цинковые покрытия относятся к анодным и защищают стальные поверхности электрохимически. В атмосферных условиях поверхность цинка тускнеет вследствие образования тонкого слоя оксида, защищающего металл от дальнейшего окисления ${ }^{1}[1,2]$.

В настоящее время для защиты от коррозии изделий из стали и чугуна применяются металлические покрытия на основе цинковых и алюминиевых сплавов, наносимые на поверхность изделий различными методами [3-6]. Наиболее универсальными и распространенными являются цинкалюминиевые покрытия типа «Гальфан-1» и «Гальфан-2» (сплавы цинка с 5 и 55\% масс. алюминия) с высокими защитными свойствами, которые можно наносить горячим методом путем погружения стальных конструкций в расплав покрывающего металла [7-9]. С целью повышения коррозионной стойкости гальфановых покрытий разработаны новые защитные цинк-алюминиевые покрытия с указанными металлами [10-12]. Имеются сведения об анодном поведении данных сплавов в кислых, нейтральных и щелочных средах [13-15]. Установлена эфффективность их легирования третьими компонентами [16-19].

В работе [20] сообщается об особенностях получения и преимуществах использования электрохимических покрытий из сплавов цинка с молибденом и оловом. Исследованы особенности нанесения покрытий Zn-Mo и Zn-Sn и сплавов на основе каждого из этих металлов. Выяв- лено, что такие покрытия имеют преимущества по сравнению с покрытиями, образованными только одним из металлов. Введение небольшого количества молибдена в состав цинкового покрытия в процессе электролиза дает возможность получать покрытия Zn-Мо-сплавов, которые отличаются более высокой защитной способностью, чем только цинковые покрытия [21]. Показана эффрективность их использования в атмосферных условиях повышенной жесткости (морские среды, приморские зоны, тропики и другие фракторы внешней среды) [20].

В научной литературе и в сети Интернет нами не обнаружено сведений, относящихся к влиянию молибдена на анодное поведение эвтектоидного сплава Zn0.5Al. Исходя из этого в качестве легирующего компонента сплава Zn0.5Al был выбран молибден.

Цель настоящей работы заключалась в исследовании влияния добавок молибдена на анодное поведение сплава Zn0.5Al и разработке оптимального состава сплавов, которые могут использоваться в качестве эффективных покрытий и протекторов для повышения коррозионной стойкости стальных конструкций, изделий и сооружений.

\section{ЭКСПЕРИМЕНТАЛЬНАЯ ЧАСТЬ}

При изучении анодного поведения сплавов давно и продуктивно используются электрохимические методы исследования, особенно потенциодинамические ${ }^{2}[22,23]$. Применяя потенциодинамические режимы исследования, можно оценить в поведении анодного сплава роль электродного потенциала в пассивном состоянии. Известно, что важнейшей анодной характе-

${ }^{1}$ Шлугер А.М., Ажогин Ф.Ф., Ефимов Е.А. Коррозия и защита металлов: учеб. пособие для металлургических специальностей вузов. М.: Металлургия. 1981. 216 с.

${ }^{2}$ Слэндер С.Д., Бойд У.К. Коррозионная стойкость цинка: справочник / пер с англ. Е.В. Проскурина, А.И. Литвиновой. М.: Металлургия. 1976. 200 с. 
Обидов З.Р., Иброхимов П.Р., Рахимов Ф.А., Ганиев И.Н. Анодное поведение сплава Zn0.5Al, ... Obidov Z.R., Ibrohimov P.R., Rahimov F.A., Ganiev I.N. Anodic behaviour of Zn0.5Al ...

ристикой процесса является зависимость кинетики растворения защитного покрытия от потенциала, важная для избрания способа защиты и повышения устойчивости анодного сплава в заданных условиях эксплуатации.

Образцы сплава для исследования были получены из цинка марки х.ч. (гранулированный), алюминия марки A7 и его лигатуры с молибденом (2\% Мо) сплавлением в тиглях из оксида алюминия в шахтной печи электрического сопротивления типа СШОЛ в интервале температур $650-750{ }^{\circ} \mathrm{C}$. Химический анализ компонентов состава исследуемых сплавов контролировался микрорентгеноспектральным анализом на приборе SEM серии AIS 2100 (Южная Корея). Из полученных сплавов в графитовую изложницу отливали стержни диаметром 8 мм и длиной 140 мм. Боковые части образцов покрывались коррозионностойким лаком. Рабочей поверхностью служил торец электрода.

Потенциодинамическое исследование анодного поведения сплава Zn0.5Al, легированного молибденом, проводили в кислых $(0,1 \mathrm{M}, \mathrm{pH}=1$; $0,01 \mathrm{M}, \mathrm{pH}=2 ; 0,001 \mathrm{M}, \mathrm{pH}=3)$, нейтральных $(0,03 ; 0,3 ; 3 \%, \mathrm{pH}=7)$ и щелочных $(0,001 \mathrm{M}$, $\mathrm{pH}=10 ; 0,01 \mathrm{M}, \mathrm{pH}=11 ; 0,1 \mathrm{M}, \mathrm{pH}=12)$ средах электролитов $\mathrm{HCl}, \mathrm{NaCl}$ и $\mathrm{NaOH}$. Скорость развертки электродного потенциала на приборе потенциостат ПИ-50.1.1 составляла 2 мВ/с. Электродом сравнения служил хлорсеребряный (х.с.э.), вспомогательным - платиновый. Методика электрохимического исследования сплавов подробно описана в работах 2 [22, 24-26]. Схематическое изображение последовательности операций при снятии поляризационных кривых образцов исследуемых сплавов, на примере сплава Zn0.5Al, легированного 0,05\% масс. молибдена, представлено на рис. 1. При коррозионно-

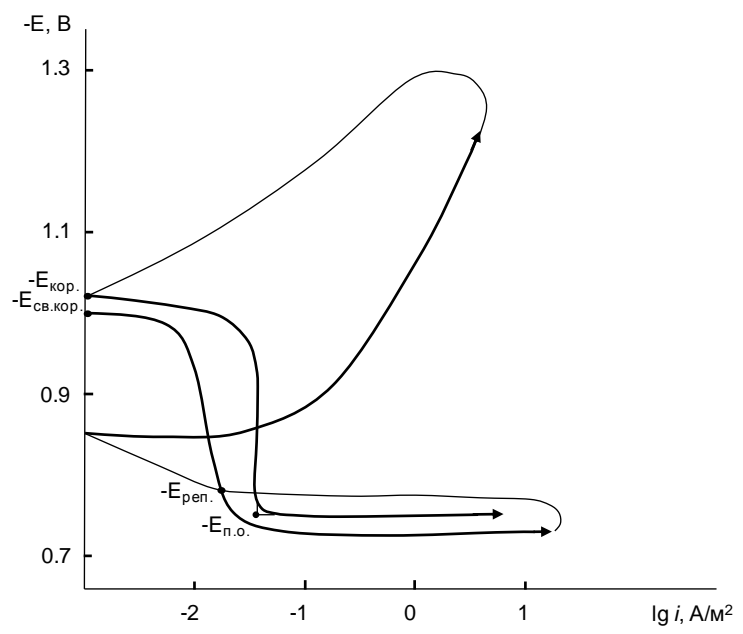

Рис. 1. Потенциодинамические анодные и катодные поляризационные кривые сплава Zn0.5Al, содержащего 0,05\% масс. молибдена, в 3\%-м электролите $\mathrm{NaCl}$

Fig. 1. Potentiodynamical anodic and cathodic polarization curves of $\mathrm{Zn0.5Al}$ alloy containing $0.05 \%$ wt. of molybdenum, in $3 \% \mathrm{NaCl}$ electrolyte электрохимических исследованиях образцы потенциодинамически поляризовали в положительном направлении от стационарного потенциала свободной коррозии $\left(-E_{c в . к о р ., ~} B\right)$, установившегося при погружении, до резкого возрастания тока в результате питтингообразования $\left(-E_{\text {п.о. }}, B\right)$. Затем образцы поляризовали в обратном направлении, в результате чего происходило подщелачивание приэлектродного слоя поверхности сплава (-E $\left.E_{\text {реп., }}-E_{\text {кор., }} B\right)$. Наконец, образцы поляризовали в положительном направлении $\left(-E_{\text {п.о. }}, B\right)$ и получали потенциодинамические анодные и катодные поляризационные кривые исследуемых сплавов (см. рис. 1). Далее по кривым определяли коррозионно-электрохимические потенциалы сплава Zn0.5Al, легированного от 0,01 до 1,0\% масс. молибдена.

\section{ОБСУЖДЕНИЕ РЕЗУЛЬТАТОВ}

Зависимость потенциала свободной коррозии сплавов от времени играет важную роль в установлении пассивности поверхности в различных средах. Результаты исследования по установлению стационарного значения потенциала свободной коррозии сплава Zn0.5Al, легированного молибденом, наблюдали в течение 1 ч. Для всех исследованных групп сплавов, содержащих 0,01-1,0\% масс. молибдена (на примере указанной среды), в первые 15 мин наблюдения отмечено незначительное смещение потенциала в область положительных значений, что объясняется процессом формирования защитной оксидной пленки, который заканчивается через 3040 мин от начала (табл. 1, рис. 2).

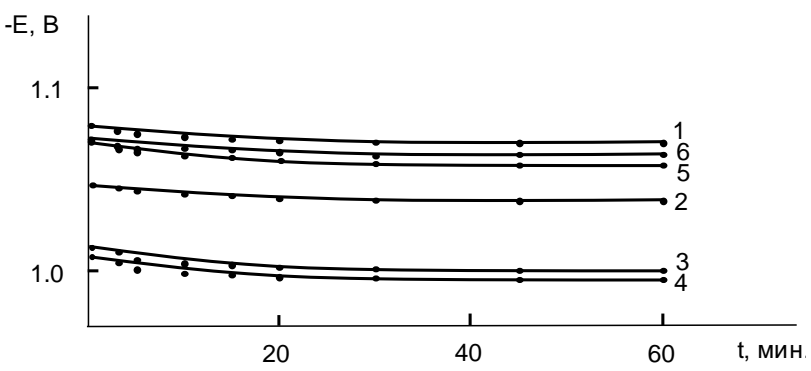

Рис. 2. Изменение потенциала свободной коррозии во времени сплава Zn0.5Al (1), содержащего молибдена, \% масс.: 0,01 (2); 0,05 (3); 0,1 (4); 0,5 (5); 1,0 (6), в среде $3 \%-$-г $\mathrm{NaCl}$

Fig. 2. Time change of free corrosion potential of Zn0.5Al (1) alloy, containing molybdenum, \% wt.: 0.01 (2); 0.05 (3); 0.1 (4); 0.5 (5); 1.0 (6) in $3 \% \mathrm{NaCl}$

В табл. 2 представлены потенциалы корро-

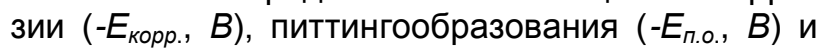
репассивации (-E $\left.E_{\text {реп., }} B\right)$ сплава Zn0.5Al, легированного молибденом, в кислых, нейтральных и щелочных средах. Видно, что добавки молибдена в пределах изученного диапазона способствуют смещению коррозионно-электрохимичес- 
Обидов З.Р., Иброхимов П.Р., Рахимов Ф.А., Ганиев И.Н. Анодное поведение сплава Zn0.5Al, ... Obidov Z.R., Ibrohimov P.R., Rahimov F.A., Ganiev I.N. Anodic behaviour of Zn0.5Al ...

ких потенциалов эвтектоидного сплава Zn0.5Al в область отрицательных значений. В результате снижается плотность тока коррозии и скорость коррозии исходного цинк-алюминиевого сплава Zn0.5Al в 1,0-2,5 раза (рис. 3).

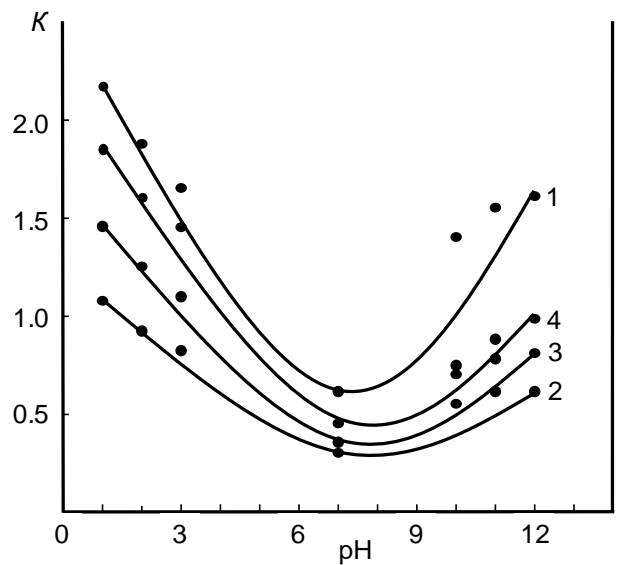

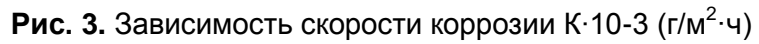
сплава Zn0.5Al (1), содержащего 0,01 (2), 0,1 (3) и 1,0\% масс. (4) молибдена от $\mathrm{pH}$ среды

Fig. 3. Corrosion rate $\mathrm{K} \cdot 10^{-3}\left(\mathrm{~g} / \mathrm{m}^{2} \mathrm{~h}\right)$ of the $\mathrm{ZnO} 0.5 \mathrm{Al}$ (1) alloy containing (2) 0.01 , (3) 0.1 , and $1.0 \mathrm{wt} \%$. (4) of molybdenum versus $\mathrm{pH}$
Введение 0,5 и 1,0\% масс. легирующего компонента в исходный сплав Zn0.5Al заметно сдвигает электрохимические потенциалы репассивации, питтингообразования и коррозии исходного сплава в отрицательном направлении. При этом скорость коррозии сплавов несколько растет, но по абсолютной величине последнее (добавка $1.0 \%$ масс. Мо) не превышает скорости коррозии исходного сплава. Следовательно, добавки молибдена в пределах 0,01-0,1\% масс. снижают скорость коррозии сплава Zn0.5Al в 2,0-2,5 раза. Более того, указанные составы сплавов наиболее устойчивы к питтинговой коррозии, особенно в кислой $(0.001 \mathrm{M}$ $\mathrm{HCl})$, нейтральной $(0,03 ; 0,3 ; 3 \% \mathrm{NaCl})$ и щелочной (0,001 М NaOH) средах (см. табл. 2, рис. 3).

Изменение скорости коррозии сплава Zn0.5Al, легированного молибденом, можно наблюдать в кислых, нейтральных и щелочных средах электролитов $\mathrm{HCl}, \mathrm{NaCl}$ и $\mathrm{NaOH}$ при различных значениях $\mathrm{pH}$ среды. Добавки молибдена в пределах 0,01-0,1\% масс. снижают скорость коррозии сплава Zn0.5Al в 2,0-2,5 раза. Повышение содержания легирующего компонента до 1,0\% масс. ее несколько увеличивает как в кислой среде, так и в щелочной. Наименьшие значения скорости коррозии исходного сплава Zn0.5Al c молибденом зафриксированы в интервале $\mathrm{pH}=5-9$ (см. рис. 3).

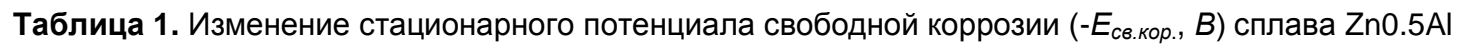
с молибденом во времени в кислой и щелочной средах

Table 1. Time changes in the free corrosion stationary potential (- $E_{s v}$ corr., $V$ ) of $\mathrm{Zn} 0.5 \mathrm{Al}$ alloy with molybdenum in acidic and alkaline ambience

\begin{tabular}{|c|c|c|c|c|c|c|c|c|c|}
\hline \multirow{2}{*}{ Среда } & \multirow{2}{*}{$\begin{array}{c}\text { Добавки Мо } \\
\text { в сплаве, } \\
\text { \% масс. }\end{array}$} & \multicolumn{8}{|c|}{ Время выдержки образцов, мин } \\
\hline & & $1 / 3$ & $2 / 3$ & 1 & 5 & 15 & 40 & 50 & 60 \\
\hline \multirow{6}{*}{$\begin{array}{c}0,01 \mathrm{M} \\
\mathrm{HCl}\end{array}$} & - & 1,123 & 1,122 & 1,117 & 1,115 & 1,111 & 1,110 & 1,110 & 1,110 \\
\hline & 0,01 & 1,146 & 1,144 & 1,141 & 1,136 & 1,125 & 1,124 & 1,124 & 1,124 \\
\hline & 0,05 & 1,155 & 1,154 & 1,152 & 1,147 & 1,134 & 1,133 & 1,133 & 1,133 \\
\hline & 0,1 & 1,168 & 1,167 & 1,165 & 1,155 & 1,148 & 1,147 & 1,147 & 1,147 \\
\hline & 0,5 & 1,191 & 1,191 & 1,189 & 1,173 & 1,166 & 1,165 & 1,165 & 1,165 \\
\hline & 1,0 & 1,203 & 1,200 & 1,197 & 1,186 & 1,175 & 1,174 & 1,174 & 1,174 \\
\hline \multirow{6}{*}{$\begin{array}{l}0,01 \mathrm{M} \\
\mathrm{NaOH}\end{array}$} & - & 1,056 & 1,055 & 1,050 & 1,050 & 1,049 & 1,048 & 1,048 & 1,048 \\
\hline & 0,01 & 1,066 & 1,066 & 1,065 & 1,062 & 1,060 & 1,060 & 1,060 & 1,060 \\
\hline & 0,05 & 1,092 & 1,091 & 1,089 & 1,089 & 1,086 & 1,085 & 1,085 & 1,085 \\
\hline & 0,1 & 1,114 & 1,112 & 1,110 & 1,105 & 1,101 & 1,099 & 1,099 & 1,099 \\
\hline & 0,5 & 1,149 & 1,146 & 1,143 & 1,139 & 1,131 & 1,126 & 1,126 & 1,126 \\
\hline & 1,0 & 1,182 & 1,179 & 1,174 & 1,168 & 1,160 & 1,157 & 1,157 & 1,157 \\
\hline
\end{tabular}

Таблица 2. Анодные характеристики (х.с.э.) сплава Zn0.5Al, легированного молибденом, в кислых, нейтральных и щелочных средах

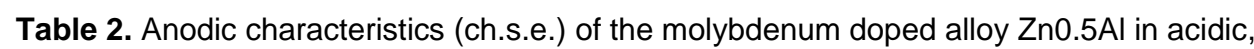
neutral and alkaline ambiences

\begin{tabular}{|c|c|c|c|c|c|c|c|}
\hline \multirow[b]{2}{*}{ Среда } & \multirow{2}{*}{$\begin{array}{c}\text { Содержание } \\
\text { Мо в сплаве, } \\
\text { \% масс. }\end{array}$} & \multicolumn{4}{|c|}{ Электрохимические потенциалы, В (х.с.э.) } & \multicolumn{2}{|c|}{ Скорость коррозии } \\
\hline & & $-E_{\text {св.кор. }}$ & $-E_{\text {кор. }}$ & $-E_{\text {п.о. }}$ & $-E_{\text {реп. }}$ & $i_{\text {кор. }} \cdot 10^{-2}, \mathrm{~A} / \mathrm{M}^{2}$ & $\mathrm{~K} \cdot 10^{-3}, \mathrm{r} / \mathrm{M}^{2} \mathrm{u}$ \\
\hline \multirow{6}{*}{$\begin{array}{c}0.001 \mathrm{M} \\
\mathrm{HCl}\end{array}$} & - & 1,045 & 1,051 & 0,912 & 0,921 & 0,136 & 1,65 \\
\hline & 0,01 & 1,057 & 1,060 & 0,928 & 0,933 & 0,068 & 0,83 \\
\hline & 0,05 & 1,064 & 1,065 & 0,935 & 0,942 & 0,074 & 0,90 \\
\hline & 0,1 & 1,079 & 1,083 & 0,952 & 0,965 & 0,090 & 1,10 \\
\hline & 0,5 & 1,093 & 1,096 & 0,968 & 0,973 & 0,105 & 1,28 \\
\hline & 1,0 & 1,108 & 1,114 & 0,975 & 0,988 & 0,118 & 1,44 \\
\hline
\end{tabular}


Обидов З.Р., Иброхимов П.Р., Рахимов Ф.А., Ганиев И.Н. Анодное поведение сплава Zn0.5Al, ... Obidov Z.R., Ibrohimov P.R., Rahimov F.A., Ganiev I.N. Anodic behaviour of Zn0.5AI ...

\begin{tabular}{|c|c|c|c|c|c|c|c|}
\hline \multirow{6}{*}{$\begin{array}{c}0,03 \% \\
\mathrm{NaCl}\end{array}$} & - & 0,960 & 0,968 & 0,745 & 0,759 & 0,037 & 0,45 \\
\hline & 0,01 & 0,922 & 0,937 & 0,738 & 0,755 & 0,019 & 0,23 \\
\hline & 0,05 & 0,910 & 0,926 & 0,715 & 0,722 & 0,018 & 0,22 \\
\hline & 0,1 & 0,900 & 0,905 & 0,700 & 0,705 & 0,023 & 0,28 \\
\hline & 0,5 & 0,953 & 0,961 & 0,740 & 0,752 & 0,029 & 0,35 \\
\hline & 1,0 & 0,957 & 0,958 & 0,742 & 0,762 & 0,031 & 0,37 \\
\hline \multirow{6}{*}{$\begin{array}{c}0,001 \mathrm{M} \\
\mathrm{NaOH}\end{array}$} & - & 1,025 & 1,033 & 0,845 & 0,853 & 0,116 & 1,41 \\
\hline & 0,01 & 1,034 & 1,045 & 0,863 & 0,880 & 0,045 & 0,55 \\
\hline & 0,05 & 1,052 & 1,066 & 0,887 & 0,899 & 0,057 & 0,69 \\
\hline & 0,1 & 1,077 & 1,082 & 0,902 & 0,907 & 0,058 & 0,71 \\
\hline & 0,5 & 1,106 & 1,117 & 0,934 & 0,947 & 0,060 & 0,73 \\
\hline & 1,0 & 1,129 & 1,140 & 0,955 & 0,970 & 0,061 & 0,74 \\
\hline \multirow{6}{*}{$\begin{array}{c}0,1 \mathrm{M} \\
\mathrm{HCl}\end{array}$} & - & 1,190 & 1,195 & 1,030 & 1,036 & 0,178 & 2,17 \\
\hline & 0,01 & 1,204 & 1,210 & 1,040 & 1,045 & 0,089 & 1,09 \\
\hline & 0,05 & 1,211 & 1,215 & 1,045 & 1,057 & 0,096 & 1,17 \\
\hline & 0,1 & 1,215 & 1,215 & 1,053 & 1,064 & 0,120 & 1,46 \\
\hline & 0,5 & 1,237 & 1,240 & 1,068 & 1,078 & 0,137 & 1,67 \\
\hline & 1,0 & 1,244 & 1,248 & 1,075 & 1,082 & 0,151 & 1,84 \\
\hline \multirow{6}{*}{$3,0 \%$} & - & 1,070 & 1,086 & 0,779 & 0,794 & 0,055 & 0,67 \\
\hline & 0,01 & 1,039 & 1,048 & 0,764 & 0,785 & 0,028 & 0,34 \\
\hline & 0,05 & 1,000 & 1,020 & 0,750 & 0,780 & 0,027 & 0,33 \\
\hline & 0,1 & 0,991 & 0,996 & 0,718 & 0,723 & 0,032 & 0,39 \\
\hline & 0,5 & 1,058 & 1,064 & 0,805 & 0,811 & 0,039 & 0,47 \\
\hline & 1,0 & 1,063 & 1,075 & 0,820 & 0,827 & 0,041 & 0,50 \\
\hline \multirow{6}{*}{$\begin{array}{c}0,1 \mathrm{M} \\
\mathrm{NaOH}\end{array}$} & - & 1,210 & 1,216 & 0,920 & 0,936 & 0,133 & 1,62 \\
\hline & 0,01 & 1,215 & 1,218 & 0,930 & 0,934 & 0,052 & 0,63 \\
\hline & 0,05 & 1,219 & 1,220 & 0,967 & 0,980 & 0,055 & 0,67 \\
\hline & 0,1 & 1,238 & 1,244 & 0,989 & 0,995 & 0,068 & 0,83 \\
\hline & 0,5 & 1,269 & 1,272 & 1,000 & 1,011 & 0,075 & 0,91 \\
\hline & 1,0 & 1,276 & 1,280 & 1,110 & 1,128 & 0,080 & 0,97 \\
\hline
\end{tabular}

\section{ЗАКЛЮЧЕНИЕ}

Таким образом, легирование используемого для защиты от коррозии сплава Zn0.5Al молибденом придает защитному покрытию большую анодную устойчивость в кислой, нейтральной и щелочной средах. Эффрективное торможение той или иной стадии процесса коррозии и разрушения поверхности связано с особенностями протекания анодного процесса (например, торможения анодного процесса из-за наступления пассивного состояния). Плотность тока и скорость коррозии эвтектоидного сплава Zn0.5Al уменьшается в 2,0-2,5 раза при введении в него не более $0,1 \%$ масс. молибдена. Увеличение содержания легирующего элемента способствует исчезновению достигнутого эфрфекта и приближению свойств легированного сплава к свойствам образца (исходного слава). Отмеченный эфрфект оказывается наиболее значим в среде, близкой к нейтральной.

Более того, найденные в результате опытов некоторые оптимальные условия (по содержанию легирующего компонента и кислотности среды) важны для разработки анодных покрытий и протекторов для защиты от коррозии конструкций и изделий из стали и чугуна.

\section{СПИСОК ЛИТЕРАТУРЫ}

1. Виткин А.И., Тейндл И.И. Металлические покрытия листовой и полосовой стали. М.: Металлургия. $1971.493 \mathrm{c.}$

2. Кечин В.А., Люблинский Е.Я. Цинковые сплавы. М.: Металлургия. 1986. 247 с.

3. Mazilkin A.A., Straumal B.B., Borodachenkova M.V., Valiev R.Z., Kogtenkova O.A., Baretzky B. Gradual softening of Al-Zn alloys during highpressure torsion // Materials Letters. 2012. Vol. 84. P. 63-65. https://doi.org/10.1016/j.matlet. 2012.06.026

4. Obidov Z.R. Thermophysical properties and thermodynamic functions of the beryllium, magnesium and praseodymium alloyed Zn-55Al alloy // High Temperature. 2017. Vol. 55. Issue 1. P. 150-153. https://doi.org/10.7868/S0040364417010161

5. Яковлева А.А., Анциферов Е.А., Гусева Е.А., Садловский С.В. Влияние защитного по- крытия на основе органического связующего на коррозионную устойчивость стали // Известия вузов. Прикладная химия и биотехнология. 2019. T. 9. N 4. C. 600-611. https://doi.org/10.21285/22272925-2019-9-4-600-611

6. Valiev R.Z., Murashkin M.Yu., Kilmametov A., Straumal B., Chinh N.Q., Langdon T.G. Unusual super-ductility at room temperature in an ultrafinegrained aluminum alloy // Journal of Materials Science. 2010. Vol. 45. Issue 17. P. 4718-4724. https://doi.org/10.1007/s10853-010-4588-z

7. Najafabadimini R.A., Irani M., Ganiev I., Obidov Z. Galfan I and Galfan II doped with calcium, corrosion resistant alloys // Oriental Journal of Chemistry. 2014. Vol. 30. Issue 3. P. 969-973. https://doi. org/10.13005/ojc/300307

8. Tokuteru U., Yorinobu T., Kawasaki M., 
Обидов З.Р., Иброхимов П.Р., Рахимов Ф.А., Ганиев И.Н. Анодное поведение сплава Zn0.5Al, ... Obidov Z.R., Ibrohimov P.R., Rahimov F.A., Ganiev I.N. Anodic behaviour of Zn0.5Al ...

Higashi K. Achieving room-temperature superplasticity in an ultrafin-grainer $\mathrm{Zn}-22 \%$ Al alloy // Letters on Materials. 2015. Vol. 5. Issue 3 (19). P. 269-275. https://doi.org/10.22226/2410-3535-2015-3-269-275

9. Обидов 3.Р. Анодное поведение и окисление сплавов Zn5Al и Zn55Al, легированных барием // Известия Санкт-Петербургского государственного технологического института (технического университета). 2015. N 31 (57). С. 51-54.

10. Amini R., Obidov Z., Ganiev I., Razazi M. Potentiodynamical research of $\mathrm{Zn}-\mathrm{Al}-\mathrm{Mg}$ alloy system in the neutral ambience of $\mathrm{NaCl}$ electrolyte and influence of $\mathrm{Mg}$ on the structure // Journal of Surface Engineered Materials and Advanced Technology. 2012. Vol. 2. Issue 2. P. 110-114. https://doi.org/10.4236/ jsemat.2012.22017

11. Gurjar M.S., Dehiya S., Sharma M., Upadhyay N.C. Effect of fly ash particles on the mechanical properties of $\mathrm{Zn}-22 \%$ Al alloy via stir castimg method // IOSR Journal of Mechanical and Civil Engineering. 2013. Vol. 10. Issue 2. P. 39-42.

12. Obidov Z.R. Effect of $\mathrm{pH}$ on the anodic behavior of beryllium and magnesium doped alloy Zn55Al // Russian Journal of Applied Chemistry. 2015. Vol. 88. Issue 9. P. 1451-1457. https://doi.org/ 10.1134/S1070427215090116

13. Amini R., Obidov Z., Ganiev I., Razazi M. Anodic behavior of $\mathrm{Zn}$-Al-Be alloys in the $\mathrm{NaCl}$ solution and the influence of $\mathrm{Be}$ on structure // Journal of Surface Engineered Materials and Advanced Technology. 2012. Vol. 2. Issue 2. P. 127-131. https://doi.org/10.4236/jsemat.2012.22020

14. Обидов 3.Р. Влияние $\mathrm{pH}$ среды на анодное поведение сплава Zn5Al, легированного бериллием и магнием // Известия Санкт-Петербургского государственного технологического института (технического университета). 2015. N 32 (58). C. $43-46$.

15. Obidov Z.R., Amonova A.V., Ganiev I.N. Influence of the $\mathrm{pH}$ of the medium on the anodic behavior of scandium - doped Zn55Al alloy // Russian Journal of Non-Ferrous Metals. 2013. Vol. 54. Issue 3. P. 234238. https://doi.org/10.3103/S1067821213030115

16. Lin K.L., Yang C.F., Lee J.T. Correlation of microstructure with corrosion and electrochemical behaviours of the bach-type hot-dip Al-Zn coatings: Part 1. Zn and 5\% Al-Zn coatings // Corrosion. 1991. Vol. 47. Issue 1. P. 9-17. https://doi.org/10.5006/ 1.3585224

17. Obidov Z.R., Amonova A.V., Ganiev I.N. Effect of scandium doping on the oxidation resistance of Zn5Al and Zn55Al alloys // Russian Journal of Physical Chemistry A. 2013. Vol. 87. Issue 4. P. 702703. https://doi.org/10.1134/S0036024413040201

18. Lin K.L., Yang C.F., Lee J.T. Correlation of microstructure with corrosion and electrochemical behaviours of the bach-type hot-dip Al-Zn coatings: Part 2. $55 \%$ Al-Zn coatings // Corrosion. 1991. Vol. 47. Issue 1. P. 17-23. https://doi.org/10.5006/1.3585213

19. Obidov Z.R. Anodic behavior and oxidation of strontium-doped Zn5Al and Zn55Al alloys // Protection of Metals and Physical Chemistry of Surfaces. 2012. Vol. 48. Issue 3. P. 352-355. https://doi.org/ 10.1134/S2070205112030136

20. Герасименко А.А. Об особенностях получения и преимуществах использования электрохимических покрытий сплавами цинка с оловом и молибденом // Технологии в электронной промышленности. 2010. N 7. С. 33-39.

21. Атрашкова В.В., Атрашков В.К., Герасименко А.А. Осаждение цинк-молибденовых покрытий // Защита металлов. 1995. Т. 31. N 3. C. $67-71$.

22. Фрейман Л.И., Макаров В.А., Брыксин И.Е. Потенциостатические методы В коррозионных исследованиях и электрохимической защите. Л.: Химия. 1972. $240 \mathrm{c.}$

23. Постников Н.С. Коррозионностойкие алюминиевые сплавы. М.: Металлургия. 1976. 301 с.

24. Колотыркин Я.М. Металл и коррозия. М.: Металлургия. 1985. 88 с.

25. Семенова И.В., Флорианович Г.М., Хорошилов А.В. Коррозия и защита от коррозии. М.: Физматлит. 2002. 336 с.

26. Улиг Г.Г., Реви Р.У. Коррозия и борьба с ней. Введение в коррозионную науку и технику / пер. с англ. А.М. Сухотина, А.И. Хентова; под ред. А.М. Сухотина. Л.: Химия. 1989. 456 с.

\section{REFERENCES}

1. Vitkin Al, Teindl II. Metal coverings of a sheet and strip steel. Moscow: Metallurgiya; 1971. 493 p. (In Russian)

2. Kechin VA, Lyblinskii EYa. Zinc alloys. Moscow: Metallurgiya; 1986. 247 p. (In Russian)

3. Mazilkin AA, Straumal BB, Borodachenkova MV, Valiev RZ, Kogtenkova OA, Baretzky B. Gradual softening of Al-Zn alloys during highpressure torsion. Materials Letters. 2012;84:63-65. https://doi.org/10.1016/j.matlet. 2012.06.026

4. Obidov ZR. Thermophysical properties and thermodynamic functions of the beryllium, magnesium and praseodymium alloyed $\mathrm{Zn}-55 \mathrm{Al}$ alloy. High Temperature. 2017;55(1):150-153. https://doi.org/

\subsection{8/S0040364417010161}

5. Yakovleva AA, Antsiferov EA, Guseva EA, Sadlovsky SV. Effect of an organic binder protective coating on the corrosion resistance of steel. Izvestiya Vuzov. Prikladnaya Khimiya $i$ Biotekhnologiya $=$ Proceedings of Universities. Applied Chemistry and Biotechnology. 2019;9(4):600-611. (In Russian) https:// doi.org/10.21285/2227-2925-2019-9-4-600-611

6. Valiev RZ, Murashkin MYu, Kilmametov A, Straumal B, Chinh NQ, Langdon TG. Unusual superductility at room temperature in an ultrafine-grained aluminum alloy. Journal of Materials Science. 2010;45(17):4718-4724. https://doi.org/10.1007/ s10853-010-4588-z 
Обидов З.Р., Иброхимов П.Р., Рахимов Ф.А., Ганиев И.Н. Анодное поведение сплава Zn0.5Al, ... Obidov Z.R., Ibrohimov P.R., Rahimov F.A., Ganiev I.N. Anodic behaviour of Zn0.5Al ...

7. Najafabadimini RA, Irani M, Ganiev I, Obidov Z. Galfan I and Galfan II doped with calcium, corrosion resistant alloys. Oriental Journal of Chemistry. 2014;30(3)969-973. https://doi.org/10.13005/ojc/ 300307

8. Tokuteru U, Yorinobu T, Kawasaki M, Higashi K. Achieving room-temperature superplasticity in an ultrafin-grainer $\mathrm{Zn}-22 \% \mathrm{Al}$ alloy. Letters on Materials. 2015;5(3):269-275. https://doi.org/10.22226/24 10-3535-2015-3-269-275

9. Obidov $Z$. Anodic behavior and oxidation of the Zn5Al and Zn55Al alloy, doped with barium. Izvestiya Sankt-Peterburgskogo gosudarstvennogo tekhnologicheskogo instituta (tekhnicheskogo universiteta) = Bulletin of the Saint Petersburg State Institute of Technology (Technical University). 2015;31:51-54. (In Russian)

10. Amini R, Obidov Z, Ganiev I, Razazi M. Potentiodynamical research of $\mathrm{Zn}-\mathrm{Al}-\mathrm{Mg}$ alloy system in the neutral ambience of $\mathrm{NaCl}$ electrolyte and influence of Mg on the structure. Journal of Surface Engineered Materials and Advanced Technology. 2012;2(2):110-114. https://doi.org/10.4236/jsemat.20 12.22017

11. Gurjar MS, Dehiya S, Sharma M, Upadhyay NC. Effect of fly ash particles on the mechanical properties of $\mathrm{Zn}-22 \% \mathrm{Al}$ alloy via stir casting method. IOSR Journal of Mechanical and Civil Engineering. 2013;10(2):39-42.

12. Obidov ZR. Effect of $\mathrm{pH}$ on the anodic behavior of beryllium and magnesium doped alloy Zn55Al. Russian Journal of Applied Chemistry. 2015;88(9): 1451-1457. https://doi.org/10.1134/S10704272150 90116

13. Amini R, Obidov Z, Ganiev I, Razazi M. Anodic behavior of $\mathrm{Zn}-\mathrm{Al}-\mathrm{Be}$ alloys in the $\mathrm{NaCl}$ solution and the influence of Be on structure. Journal of Surface Engineered Materials and Advanced Technology. 2012;2(2):127-131. https://doi.org/10.4236/jsemat.2012.22020

14. Obidov $\mathrm{ZR}$. Influence of $\mathrm{pH}$ of a medium on anodic behavior of Zn5Al alloy doped with beryllium and magnesium. Izvestiya Sankt-Peterburgskogo gosudarstvennogo tekhnologicheskogo instituta (tekhnicheskogo universiteta) $=$ Bulletin of the Saint Petersburg State Institute of Technology (Technical University). 2015;32:43-46. (In Russian)

15. Obidov ZR, Amonova AV, Ganiev IN. Influence of the $\mathrm{pH}$ of the medium on the anodic behavior

\section{СВЕДЕНИЯ ОБ АВТОРАХ}

\section{Обидов Зиёдулло Рахматович,}

д.Х.н., доцент, главный научный сотрудник, Институт химии им. В.И. Никитина

АН Республики Таджикистан,

734063, г. Душанбе, ул. Айни, 299/2,

Республика Таджикистан,

凶 e-mail: z.r.obidov@rambler.ru of scandium - doped Zn55Al alloy. Russian Journal of Non-Ferrous Metals. 2013;54(3):234-238. https:// doi.org/10.3103/S1067821213030115

16. Lin KL, Yang CF, Lee JT. Correlation of microstructure with corrosion and electrochemical behaviours of the bach-type hot-dip Al-Zn coatings: Part 1. $\mathrm{Zn}$ and $5 \% \mathrm{Al}-\mathrm{Zn}$ coatings. Corrosion. 1991;47(1):9-17. https://doi.org/10.5006/1.3585224

17. Obidov ZR, Amonova AV, Ganiev IN. Effect of scandium doping on the oxidation resistance of Zn5Al and Zn55Al alloys. Russian Journal of Physical Chemistry A. 2013;87(4):702-703. https://doi.org/10. 1134/S0036024413040201

18. Lin KL, Yang CF, Lee JT. Correlation of microstructure with corrosion and electrochemical behaviors of the batch-type hot-dip Al-Zn coatings: Part 2. 55\% Al-Zn coatings. Corrosion. 1991;47(1):17-23. https://doi.org/10.5006/1.3585213

19. Obidov ZR. Anodic behavior and oxidation of strontium-doped Zn5Al and Zn55Al alloys. Protection of Metals and Physical Chemistry of Surfaces. 2012;48(3):352-355. https://doi.org/10.1134/S2070 205112030136

20. Gerasimenko AA. About features of reception and use advantages of electrochemical coverings of zinc alloys with tin and molybdenum. Tekhnologii $v$ elektronnoi promishlennosti. 2010;7:33-39. (In Russian)

21. Atrashkova VV, Atrashkov VK, Gerasimenko AA. Eltctrodipositon of zinc-molybdenum coatings. Zashchita metallov. 1995;31(3):313-314. (In Russian)

22. Freiman LI, Макаrov VA, Bryksin IE. Potentsiostatical methods in corrosion researches and electrochemical protection. Leningrad: Khimiya; 1972. 240 p. (In Russian)

23. Postnikov NS. Corrosion-resistant aluminum alloys. Moscow: Metallurgiya; 1976. 301 p. (In Russian)

24. Kolotyrkin YaM. Metal and corrosion. Moscow: Metallurgiya; 1985. 88 p. (In Russian)

25. Semenova IV, Florianovich GM, Khoroshilov AV. Corrosion and corrosion protection. Moscow: Fizmatlit; 2002. 336 p. (In Russian)

26. Uhlig HH, Revie RW. Corrosion and corrosion control. An Introduction to Corrosion Science and Engineering. 3-rd Edition. New York: John Wiley \& Sons. 1985. (Russ. ed.: Ulig GG, Revi RU. Korroziya $i$ bor'ba $s$ nei. Vvedenie $v$ korrozionnuyu nauku $i$ tekhniku. Leningrad: Khimiya; 1989. 456 p.)

\section{INFORMATION ABOUT THE AUTHORS}

\author{
Ziyodullo R. Obidov, \\ Dr. Sci. (Chemistry), Assistant Professor, \\ Chief Researcher, \\ V.I. Nikitin Institute of Chemistry, \\ National Academy of Sciences of Tajikistan, \\ 299/2, Ayni St., Dushanbe, 734063 , \\ Republic of Tajikistan, \\ 凶 e-mail: z.r.obidov@rambler.ru
}


Обидов З.Р., Иброхимов П.Р., Рахимов Ф.А., Ганиев И.Н. Анодное поведение сплава Zn0.5Al, ... Obidov Z.R., Ibrohimov P.R., Rahimov F.A., Ganiev I.N. Anodic behaviour of Zn0.5Al ...

\section{Иброхимов Пайрав Рустамович,} соискатель, Институт химии им. В.И. Никитина АН Республики Таджикистан, 734063, г. Душанбе, ул. Айни, 299/2, Республика Таджикистан

\section{Рахимов Фируз Акбарович,} старший научный сотрудник, Института химии им. В.И. Никитина АН Республики Таджикистан, 734063, г. Душанбе, ул. Айни, 299/2, Республика Таджикистан

\section{Ганиев Изатулло Наврузович,}

д.Х.Н., профрессор, академик АН Республики Таджикистан, заведующий лабораторией коррозионностойких материалов,

Институт химии им. В.И. Никитина

АН Республики Таджикистан,

734063, г. Душанбе, ул. Айни, 299/2,

Республика Таджикистан

\section{Заявленный вклад авторов}

Все авторы сделали эквивалентный вклад в подготовку публикации.

\section{Конфликт интересов}

Авторы заявляют об отсутствии конфрликта интересов.

Все авторы прочитали и одобрили окончательный вариант рукописи.

Поступила в редакцию 20.04.2021.

Одобрена после рецензирования 20.05.2021.

Принята к публикации 31.05.2021.
Pairav R. Ibrohimov,

Applicant,

V.I. Nikitin Institute of Chemistry,

National Academy of Sciences of Tajikistan, 299/2, Ayni St., Dushanbe, 734063,

Republic of Tajikistan

\section{Firuz A. Rahimov,}

Senior Scientific,

V.I. Nikitin Institute of Chemistry,

National Academy of Sciences of Tajikistan, 299/2, Ayni St., Dushanbe, 734063,

Republic of Tajikistan

\section{Izatullo N. Ganiev,}

Dr. Sci. (Chemistry), Professor, Academician of Academy of Sciences

of the Republic of Tajikistan,

Head of the Laboratory

of Corrosion-resistant Materials, V.I. Nikitin Institute of Chemistry, National Academy of Sciences of Tajikistan, 299/2, Ayni St., Dushanbe, 734063, Republic of Tajikistan

\section{Contribution of the authors}

The authors contributed equally to this article.

\section{Conflict interests}

The authors declare no conflict of interests regarding the publication of this article.

The final manuscript has been read and approved by all the co-authors.

The article was submitted 20.04.2021. Approved after reviewing 20.05.2021. Accepted for publication 31.05.2021. 